was applied for conditional length and body mass index. Associations of these measures with systolic and diastolic blood pressure at the age of 5-6 years were modeled before and after adjustment for current body mass index and height.

Results Higher weight-, length-, and bmi conditionals (expressing faster growth) were associated with higher blood pressure, with the exception of conditional growth between birth and 1 month. Adjusted for current height and body mass index, almost none of the conditionals were associated with blood pressure. However, lower birth weight and lower weight and height conditionals 0 to 1 month were associated with higher systolic blood pressure at age 5-6 years.

Conclusions We infer that the inverse association between prenatal and first month growth with childhood blood pressure may reflect a programming effect, while the positive association between infant growth after 1 month with childhood blood pressure probably reflects an effect of the tracking of body size.

\section{AETIOLOGY AND ELECTROCARDIOGRAM FINDINGS IN CHILDREN PRESENTING TO THE EMERGENCY DEPARTMENT WITH CHEST PAIN}

doi:10.1136/archdischild-2012-302724.0504

'RHK Tan, ${ }^{2} \mathrm{R}$ Saravanan, ${ }^{2} \mathrm{E}$ Ladusans, ${ }^{1} \mathrm{M}$ Ryan. ${ }^{1}$ Accident \& Emergency Department; ${ }^{2}$ Cardiology Department, Alder Hey Childrens' Hospital, Liverpool, UK

Introduction Chest pain is a frequent cause of attendance to the emergency department. Electrocardiogram (ECG) is often used as a first line investigation. This study aims to investigate the aetiology of chest pain and usefulness of ECG in a group of 200 children ranging between 3-16 years who presented between June - December 2009.

Methods ED casenotes were reviewed retrospectively to study the demographic features, clinical characteristics, ECG utilization and final diagnoses of these children. Children were placed in two groups. The first group were those who had suggestive history (crushing/exertional pain/palpitations), positive examination findings, or previous cardiac/family history. The second group of patients consisted of those without these features.

Results Musculoskeletal chest pain (46\%) is the most common diagnosis in children followed by respiratory $(15 \%)$, gastrointestinal $(14 \%)$, idiopathic $(13 \%)$, and psychological $(7.5 \%)$ causes. Cardiac cause chest pain $(4.5 \%)$ was the least common.

Only 20 patients $(10 \%)$ presented with positive cardiac features according to the criteria used. 81 (40.5\%) patients in total had an ECG performed. Of the 20 patients with cardiac features, $15(75 \%)$ had an ECG compared to $66(37 \%)$ patients in the other group. $(p<0.0014)$ Four abnormal ECGs were obtained. These were all from the group with positive cardiac features. $(p<0.0008)$.

Conclusion Musculoskeletal pain was found to be the most common cause of chest pain. Electrocardiogram has poor yield when performed in children without significant cardiac history/examination findings. However, it remains a useful screening tool and is beneficial for reassurance of patients and their families.

\section{LINEAR GROWTH IN CHILDREN WITH CONGENITAL ACYANOTIC HEART DISEASE (CHD) BEFORE VERSUS AFTER SURGICAL INTERVENTION}

doi:10.1136/archdischild-2012-302724.0505

${ }^{1} \mathrm{~A}$ Soliman, ${ }^{2} \mathrm{H}$ Yassin, ${ }^{2} \mathrm{~S}$ Saeed, ${ }^{2} \mathrm{~A}$ Khellah, ${ }^{3 \mathrm{R}}$ Elalaily, ${ }^{1} \mathrm{~A}$ Elawwa. 'Pediatrics; ${ }^{2}$ Cardiology; ${ }^{3}$ Primary Health Care, Hamad Medical Center, Doha, Oatar

Objectives To evaluate linear growth and insulin-like growth factor- I (IGF-I) concentration of patients with congenital acyanotic heart disease (CHD) before versus after surgical intervention.
Design 27 infants and children with CHD (10 with VSD, 8 with ASD, 9 PDA) without heart failure, before and 12 months or more after surgical or catheter intervention. Eighty normal children served as controls.

Results At presentation, age $=(35.6+/-26$ months $)$, patients were significantly shorter, height SDS $(\mathrm{HtSDS})(-1.6+/-1.1)$ and had lower BMI $(15.1+/-2.5)$ compared to normal controls (HtSDS = $0.25+/-0.3, \mathrm{BMI}=(16.4+/-1.5)$. One year or more after catheter or surgical treatment the HtSDS and BMI increased significantly in patients to $-0.55+/-0.9$ and $15.9+/-1.5$ respectively. Circulating concentrations of IGF-I increased from $46.8+/-29 \mathrm{mcg} / \mathrm{L}$ before to $77.3+/-47.6 \mathrm{mcg} / \mathrm{L}$ after intervention. The HtSDS after treatment was correlated with the IGF-I concentration $(r=0.804, \mathrm{P}<0.001)$. The change in the HtSDS after intervention was correlated significantly with BMI $(r=0.594, p<0.001)$. The shunt size was correlated negatively with BMI before intervention $(r=-0.35, \mathrm{P}<0.01)$ and with HtSDS $(r=-0.461, p<0.05)$.

Conclusions These data denoted that early surgical interference and good weight gain have beneficial effect on postoperative growth spurt. The accelerated linear growth after intervention appears to be mediated through activation of the GH/IGF-I system.

\section{DO SOUTH ASIAN BABIES HAVE MORE CONGENITAL HEART DEFECTS, DOES CONSANGUINITY INFLUENCE IT? FINDINGS FROM BORN IN BRADFORD STUDY}

doi:10.1136/archdischild-2012-302724.0506

'P Agadoorappa, ${ }^{2}$ S Oddie, ${ }^{3} \mathrm{~J} \mathrm{Gibbs,}{ }^{4} \mathrm{~N}$ Pawson, Born in Bradford Cohort. 'Paediatrics, Bradford Teaching Hospitals, Leeds; 'Neonatology, Bradford Teaching Hospitals, Bradford; ${ }^{3}$ Paediatric Cardiology, Leeds Teaching Hospitals NHS Trust, Leeds; ${ }^{4}$ Bradford Institute of Health Research, Bradford Teaching Hospitals, Bradford, UK

Background and Aims Born in Bradford is a birth cohort study established in March 2007 to look at health and outcomes in a multi ethnic population in Bradford (UK). We aimed to establish whether South Asian children in the cohort had a higher prevalence of congenital cardiac anomalies compared to non South Asians, and evaluate association with consanguinity.

Methods Babies with cardiac anomalies in the cohort born between May 2007 and March 2010 identified using multiple ascertainment models. Ethnicity ascribed to cases using clinical records $\&$ inferred to cohort based on questionnaire responses. Data on consanguinity and other cofounders were obtained from database. Statistical analysis done by Chi square test and logistic regression.

Results Structural congenital cardiac defects $=96$ cases. Overall prevalence rate $=8.8 / 1000$ live births. Prevalence was significantly higher in South Asians compared to non South Asians for all cardiac anomalies $(11 / 1000$ vs $6.2 / 1000, \mathrm{P}<0.05) \&$ anomalies including only VSDs requiring surgery $(8.7 / 1000$ vs $4.4 / 1000, \mathrm{P}<0.05)$ implying that the differences were unlikely due to ascertainment bias. Complex cardiac defects were more prevalent in South Asians, cyanotic defects were significantly higher in this group $\mathrm{P}=0.017$. South Asian ethnicity (Odds-ratio=1.76, $\mathrm{P}=0.019$ ) and consanguinity (Odds-ratio $=2.002, \mathrm{P}=0.003$ ) significantly increased the risk for cardiac anomalies in univariate analysis. Multivariate analysis incorporating confounding factors attenuated their effect; however consanguinity remained a borderline significant predictor.

Conclusions We have demonstrated an apparent excess of congenital cardiac anomalies in South Asians in the Born in Bradford cohort. Consanguinity seemed to increase the risk for cardiac anomalies.

\section{A NOVEL METHOD FOR QUANTIFICATION OF LEFT VENTRICULAR HYPERTRABECULATION/NONCOMPACTION USING TWO-DIMENSIONAL ECHOCARDIOGRAPHY}

doi:10.1136/archdischild-2012-302724.0507 